〈制导与对抗〉

\title{
水面舰船红外隐身总体设计分析
}

\author{
李艇, 付云鹏, 杨卫英 \\ (中国船舶及海洋工程设计研究院, 上海 200011)
}

\begin{abstract}
摘要: 隐身性能是当代舰船重要的战技指标, 直接影响舰船的生命力, 红外隐身作为舰船隐身的主 要组成部分, 是各国先进战舰隐身设计的重点之一。为研究如何提高水面舰船红外隐身性能, 对其 3 $5 \mu \mathrm{m}$ 波段的红外点源特征和 8 14 $\mu \mathrm{m}$ 波段的红外面源特征进行了分析, 并分别针对两种红外辐 射源, 对舰船常用的红外辐射抑制原理和设计方法进行了分析, 明确各种红外隐身技术如何在舰船 设计中进行应用, 可为舰船红外隐身总体设计提供参考。
\end{abstract}

关键词: 红外隐身; 隐身水幕; 排气降温; 舰船

中图分类号：U662.2 文献标识码：A 文章编号：1001-8891(2020)-02-0134-05

\section{Design Analysis of Ship Infrared Stealth}

\author{
LI Ting, FU Yunpeng, YANG Weiying \\ (Marine Design \& Research Institute of China, Shanghai 200011, China)
}

\begin{abstract}
Stealth performance is an important combat skill indicator for contemporary ships, thus affecting the vitality of a ship directly. Infrared stealth is one of the main components of ship stealth and is one of the key points of stealth design of advanced warships in various countries. In order to study how the infrared stealth performance of a ship may be improved, its infrared point source characteristics for the 3-5 $\mu \mathrm{m}$ band and its infrared face source characteristics for the 8-14 $\mu \mathrm{m}$ band are analyzed. The infrared radiation suppression principle and design method commonly used in ships are evaluated for two kinds of infrared radiation sources. The study defines how various infrared stealth technologies can be effectively applied in ship design. This research can serve as a reference for the design of infrared stealth for a ship.
\end{abstract}

Key words: infrared stealth, stealth water curtain, exhaust cooling, ships

\section{0 引言}

水面舰船作为海上武备及战机载体, 具有运载能 力强、布置灵活的优点, 大型战舰一度成为强国称霸 世界的主要工具。而随着反舰武器和探测设备的飞速 发展, 舰船的暴露和被攻击命中概率大幅度提高。因 此各海军大国开始重视舰船的隐身设计, 纷纷推出具 有高度隐身性能的先进舰船, 通过提高舰船自身的隐 蔽性, 增加防护和对抗能力 ${ }^{[1]}$ 。根据探测手段的不同, 舰船隐身技术可以分为雷达波隐身、声隐身、电场隐 身、磁场隐身以及红外隐身等。红外隐身是舰船应对 红外制导反舰武器的主要手段, 是舰船隐身性的重要
组成部分之一, 水面战斗舰艇及需要随编队伴航的辅 助舰船都对红外隐身性能提出越来越严苛的要求。

舰船研制过程中根据需要进行红外辐射的本征 控制和应急控制。本征控制即舰船红外辐射的固定控 制设计, 目的在于缩减舰船固有的红外特征; 应急控 制即临时控制设计, 当舰船需要对抗敌方红外搜索跟 踪时, 采取措施进一步缩减或改变舰船的红外特征, 以便摆脱或欺骗敌方的红外搜索跟踪。

根据红外制导原理, 舰船红外隐身的基本原则 在于设法降低舰船热辐射源的辐射强度和改变舰体 表面的辐射率。一切温度高于绝对零度的物体都在 不停辐射红外线，大气中各种气体对不同波段的红

收稿日期：2019-05-26; 修订日期：2019-07-05.

作者简介: 李艇（1980-）, 男, 四川人, 高级工程师, 主要从事舰船动力系统研究。E-mail: pzhlt_sc@sina.com。 
外辐射有不同的吸收和影响, 从而造成不同程度的 衰减。但红外线在 3 $5 \mu \mathrm{m}$ 和 8 $14 \mu \mathrm{m}$ 波段, 大气 对红外辐射基本上是透明的，被称为 “大气窗口” [2]。 红外探测器就是利用这个 “大气窗口” 对舰船进行 $3 \sim 5 \mu \mathrm{m}$ 点源识别和 $8 \sim 14 \mu \mathrm{m}$ 波段面源识别, 并引 导导弹攻击。

本文将分别分析水面舰船 3 $5 \mu \mathrm{m}$ 和 $8 \sim 14 \mu \mathrm{m}$ 两个波段红外辐射源和红外隐身设计方法, 系统阐述 主要的舰船红外抑制技术及其应用案例。

\section{1 舰船 3～5 $\mu \mathrm{m}$ 波段点源隐身设计}

舰船动力装置排气温度较高, 其 $3 \sim 5 \mu \mathrm{m}$ 波段 点源红外辐射特征主要来自排气烟羽、被加热的排气 管, 以及被烟气流加热的烟图外壁, 其中排气烟羽最 高温度可达 $500^{\circ} \mathrm{C}$, 排气管和烟图外壁被烟羽直接加 热, 温度可接近排气烟羽温度。舰船 3 5 $\mu \mathrm{m}$ 波段 点源隐身设计重点在于采取措施降低动力装置的排 烟温度以及排气管、烟图金属壁面温度。除了常规烟 图排气方式外, 为了进一步提高舰船隐身性能, 开始 采用海水喷淋舷侧排气和艉部排气技术替代传统的 烟图排气, 大幅度降低排气温度, 有效控制舰船 3 $5 \mu \mathrm{m}$ 波段红外辐射强度。

\section{1 常规烟图排气红外抑制系统设计}

\subsection{1 排气红外抑制装置}

在排气系统增设排气红外抑制装置是一种常用 的降低烟气温度设计方法, 各个国家开发了多种排气 红外抑制装置, 如 DRES 球型、引射/扩压型等。不 同型式的红外抑制装置基本原理大致相同, 图 1 为引 射/扩压型红外抑制装置原理示意图, 排气红外抑制 装置直接安装在排气管路末端, 由引射喷头、混合管 和扩压环等组成, 排气管路中烟气主流利用引射原理 将排气管周围低温空气吸入混合管并与高温烟气混 合, 以降低排烟出口温度。同时混合管中烟气通过扩 压环间的间隙继续吸收新鲜空气, 在高温烟气和金属 壁面间形成冷却气膜, 降低金属管壁温度 ${ }^{[2]}$ 。美国阿 利伯克级驱逐舰、日本日向级两栖攻击舰等舰船均采 用了排气红外抑制装置来降低烟图部位的红外辐射 特征。

必要时还可以采用海水喷雾的方式进一步降低 排气温度, 即在引射喷头出口处设置喷雾器, 向烟气 主流喷射水雾, 水雾和新鲜空气一起混入高温烟气, 利用水雾相变大量吸热的原理, 降温效果明显。美国 DDG1000 即采用了海水和空气同时对烟气进行冷 却, 提高其红外隐身性能 ${ }^{[3]}$ 。

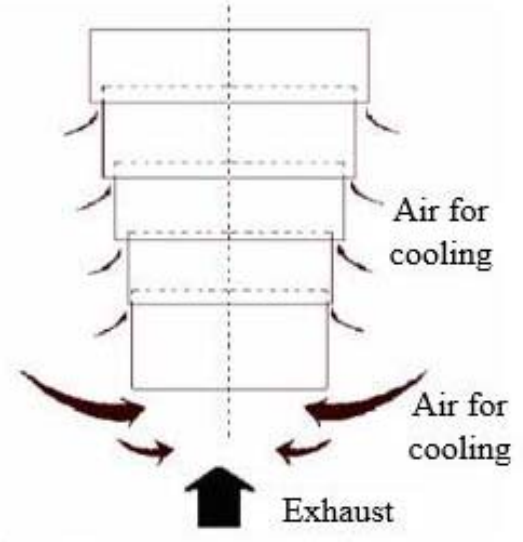

图 1 排气红外抑制装置工作原理

Fig.1 Working principle of exhaust IR suppressor

1.1.2 基于红外隐身的烟图设计

排气红外抑制装置的设置会给舰船烟图的总体 设计带来一定影响。如红外抑制装置需引入大量新鲜 空气, 因此烟囱百叶窗的进气净面积计算应考虑红外 抑制装置的引射空气量。此外百叶窗的叶片应在水平 视线内遮挡烟图内部, 防止烟图内热源 (如引射喷头) 的红外辐射通过叶片缝隙暴露出来, 即出现 “露光” 现象。

为了降低烟图壁面的二次辐射强度, 可采用烟图 壁面海水喷淋、烟囱区域气流场优化设计等方式来降 低烟图金属围壁的温度。在外板上端布置海水喷淋装 置, 如红外水幕喷头, 作为应急控制手段, 平时不开 启, 仅在作战状态或受到威胁状态时开启, 通过对烟 图金属壁板喷淋海水, 可迅速降低其温度, 彻底消除 二次辐射的亮点。图 2(a)为舰船烟图前挡板及侧板受 高温烟气影响产生二次辐射示意图, 从图中可以看 出, 烟囱侧壁板处被高温烟气最高加热至 $T_{1}$, 大大 增加了烟囱部位的红外辐射强度。对该区域进行局部 气流场的优化设计, 在烟图前挡板顶部加开百叶窗引 入新鲜空气进行降温, 烟图侧壁最高温度可降至 $T_{2}$, 如图 2(b)所示, 其中 $T_{1}$ 较 $T_{2}$ 高 $~ 60^{\circ} \mathrm{C}$ 。

针对烟图壁板的二次辐射问题, 法国拉斐特级 护卫舰的烟图采用不易于导热的玻璃钢材料进行制 造 ${ }^{[1]}$, 如图 3 所示 ${ }^{[4]}$ 。该方法有效避免了高温烟气二 次辐射产生的烟图金属围壁高温问题, 降低了全舰红 外辐射强度。

\section{2 海水喷淋舷侧排气系统设计}

舷侧排气技术在欧美等发达国家发展非常成熟， 广泛应用于游艇、商船、海洋工程、大中型水面作战 舰艇等。

海水喷淋冷却是一种相变冷却技术, 海水喷射到 排气管内后由于雾化、汽化而产生强制对流、表面蒸 
发等传热过程, 喷入的海水吸收了大量热量使得排气 温度迅速从几百摄氏度降低到几十摄氏度, 十分接近 船体表面温度, 从而可以迅速降低排气红外辐射强 度, 冷却后的烟气从舷侧排出。如图 4 所示, 海水喷 淋舷侧排气系统一般由初级消音器、喷水管段、次级

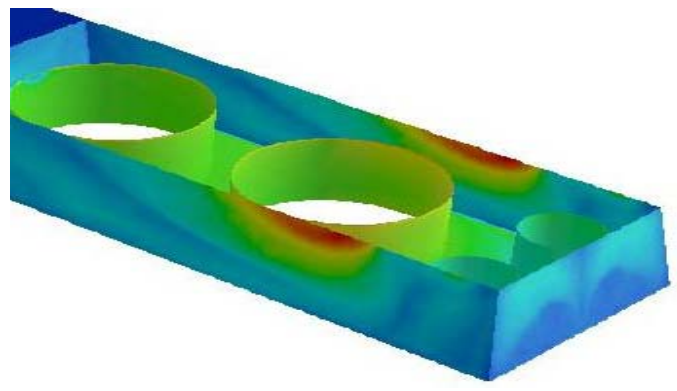

(a) 最高点温度 $T_{1}$ (a) Highest temperature: $T_{1}$

图 2 烟图部位气流场优化设计

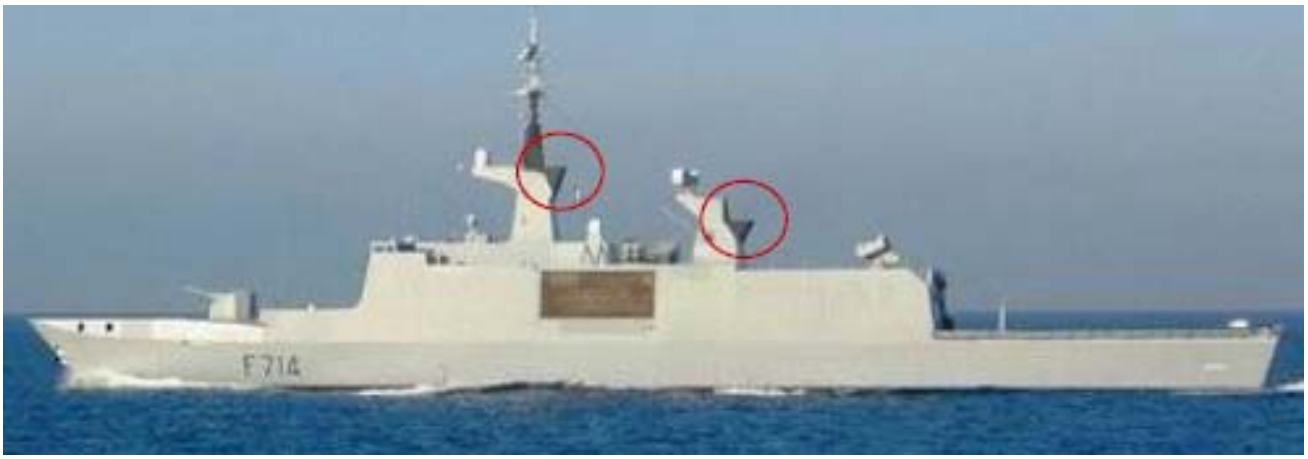

图 3 法国拉斐特级护卫舰及其烟图

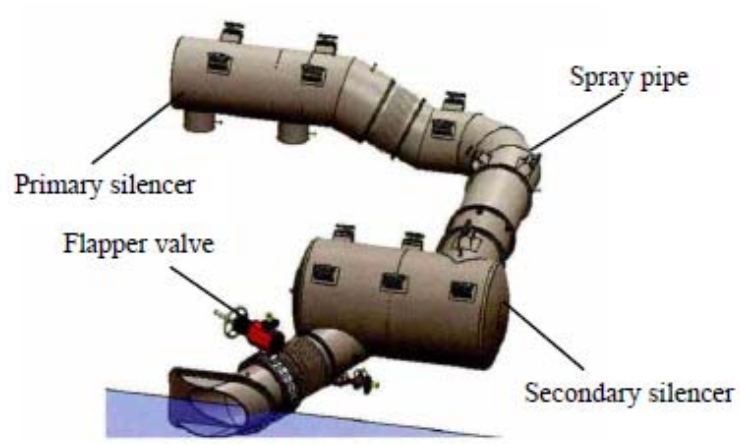

图 4 海水喷淋舷侧排气系统示意图

Fig.4 Schematic diagram of seawater spray side exhaust system

海水喷淋舷侧排气系统设计的关键问题是应采 取措施保证舷外海水不会倒灌入发动机内, 比如对于 布置靠近舷侧的发动机, 可以采用倒 U 式排管管路 布置。要对舰船䑶侧排气系统的背压进行校核计算, 同时考虑短时上浪对排气背压的影响, 系统应满足发 动机对排气背压的要求, 必要时可在满足舰船总体性 能的前提下对发动机功率进行修正。此外应对可能接 触海水的部位采取有效措施, 防止海水对排气管路及
消音器、舷侧挡板阀等组成 ${ }^{[5]}$ 。根据总体的空间布局, 也可以采用单级消音器型式。由于烟气出口温度较 低, 3 $5 \mu \mathrm{m}$ 波段红外辐射强度很小; 研究结果表明, 舷侧排气对全船 8 14 $\mu \mathrm{m}$ 波段红外辐射对比度贡献 非常小, 可忽略不计。

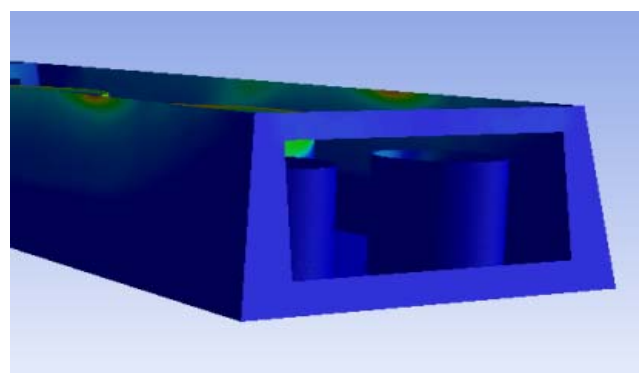

(b) 最高点温度 $T_{2}$ (b) Highest temperature: $T_{2}$

Fig.2 Optimization design of airflow field in chimney

Fig.3 French Lafayette frigate and its chimney

部件造成腐蚀。

\section{3 海水喷淋艉部排气系统设计}

海水喷淋艉部排气冷却与舷侧排气冷却原理相 似, 也是利用海水相变冷却技术, 可将高温排烟冷 却至几十摄氏度, 迅速降低排气红外辐射强度, 不 同的是冷却后的烟气从舰船艉部排出。艉部排气和 舷侧排气除了能够大幅度降低舰船的红外辐射强度 外, 可以取消传统烟图的设置, 排气管路不占用甲 板面空间, 方便武备系统的综合布置, 同时提高全 舰的雷达波隐身性能。

典型海水喷淋艉部排气系统组成如图 5 所示, 由 主排气管、应急排气管、喷淋管段、气动控制阀、应 急排气阀、挡板阀等组成。系统设计关键问题及注意 事项可参考舷侧排气系统, 在此基础上主排气管的布 置应保证舰船航行中最大纵摇角度时排气管最高处 下缘应高于水线。当舰船艉部排气管路在纵摇进水情 况下, 排气系统背压升高至设定值时, 系统控制应急 排气阀快速开启, 通过应急排气管路快速泄压, 保证 系统安全。 


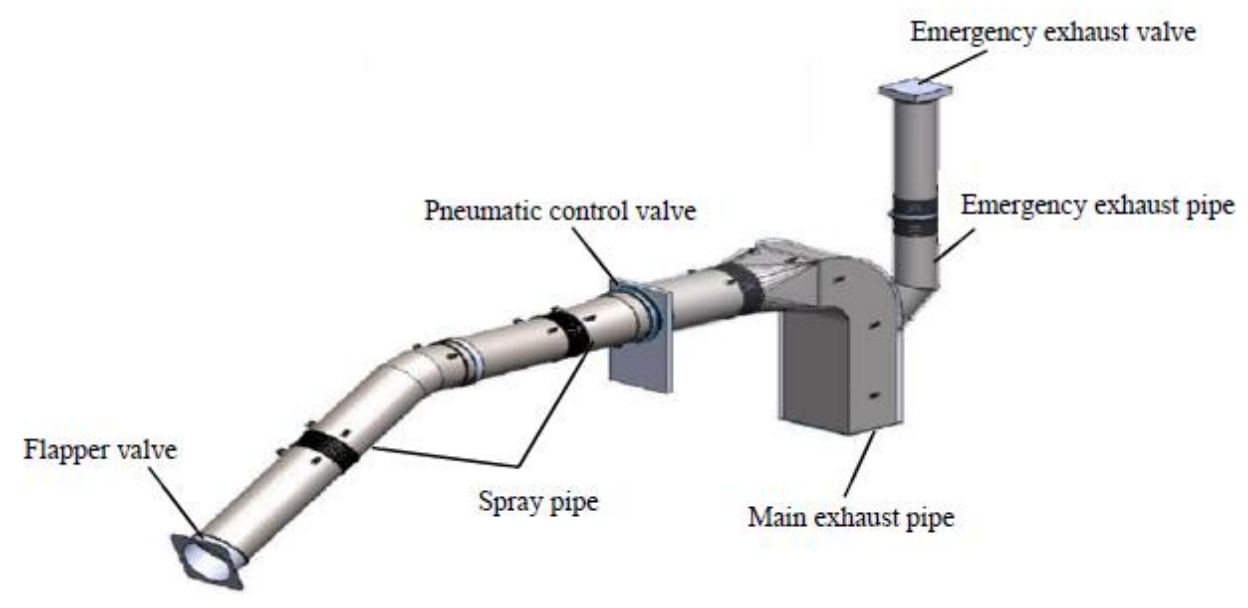

图 5 海水喷淋艉部排气系统示意图

Fig.5 Schematic diagram of seawater spray stern exhaust system

\section{2 舰船 8 14 $\mu \mathrm{m}$ 波段面源隐身设计}

研究表明, 舰船 8 14 $\mu \mathrm{m}$ 波段红外辐射特征主 要来自于船体外表面 ${ }^{[6]}$ 。因此通过降低船体表面温度 的红外水幕系统和改变船体金属表面红外发射率的 红外隐身涂料均可以作为舰船 8 14 $\mu \mathrm{m}$ 波段面源隐 身设计的有效手段。

\section{1 红外隐身水幕系统设计}

水幕系统的功能是为全舰上层建筑、露天甲板平 面和舷侧壁面提供有效的喷淋降温。在战斗需要时可 以通过水幕系统降低舰船外表面温度, 提高舰船的红 外隐身性。红外隐身水幕系统水源就近取自消防总 管, 采用遥控阀, 在消防灭火控制台的红外隐身控制 板进行控制。

随着技术的发展, 红外隐身水幕也朝着智能化、 精确控制方向发展。可通过加装红外探测器, 感知背 景的特征, 然后利用布置在船体外板的温度传感器通 过流量调节阀对喷水量进行闭环控制, 精确控制船体 与背景的红外辐射对比度。

\section{2 红外隐身涂料}

红外隐身涂料的工作原理有两种, 其中通过改变 自身涂层的发射率, 实现红外信号的调节的被称为反 射性; 吸收红外波后, 辐射的红外波会发生波长的变 化, 使波段转移, 从而不在 “大气窗口” 范围内, 称 为转换型 ${ }^{[7]}$ 。

20 世纪 70 年代后, 美国多家军事单位相继研制 出不同的红外隐身材料以及涂料, 并研发了两种不同 颜色的有机硅黏结剂的低发射率涂层。近年还提出红 外涂料的智能化与多频化, 使涂料发射率可控, 能对 背景和威胁做出迅速反应 ${ }^{[8]}$ 。同时国内也开展了大量
多波段兼容隐身材料的研究, 在一维光子晶体的基础 上进行改进，达到多波段兼容隐身的目的 ${ }^{[9]}$ 。

目前已经研发的红外隐身涂料种类较多,在应用 时需考虑其建造和维护成本, 并且要求涂料能在海上 高盐、高湿的恶劣环境下耐海水的腐蚀和冲刷，保证 使用的可靠性; 同时隐身涂料的涂装还应综合考虑雷 达波隐身和红外隐身的兼容性要求。

\section{3 结语}

红外隐身技术是舰船隐身技术的一个方面,应与 其他隐身技术整体综合地发展, 并与其他防护手段综 合考虑, 成为舰船体系防御系统中的一个有机组成部 分。本文对国内外舰船红外隐身先进技术的应用及设 计方法进行了分析, 在实船设计过程中, 可根据不同 船型特点选择合适的方法。

\section{参考文献：}

[1] 朱英富, 张国良. 舰船隐身技术[M]. 2 版: 哈尔滨: 哈尔滨工程大学 出版社, 2015.

ZHU Yingfu, ZHANG Guoliang. Ship Stealth Technology[M]. The second edition: Harbin: Harbin Engineering University Press, 2015.

[2] 徐仲方. 舰船排气红外抑制装置实验研究和数值模拟 [D]. 上海: 上 海交通大学, 2010.

XU Zhongfang. Experiment research and Numerical simulation of the Marine gas turbine IR Suppressor[D]. Shanghai: Shanghai Jiaotong University, 2010.

[3] 姜艳, 杨心武, 吴明, 等. 美海军 DDG1000 舰任务系统关键信息技 术分析[J]. 舰船电子工程, 2016(12): 14-22.

JIANG Yan, YANG Xinwu, WU Ming, et al. Key information technologies of US Navy ship DDG-1000 Mission system[J]. Ship 
Electronic Engineering, 2016(12): 14-22.

[4] 徐文. 法国的拉斐特级隐身护卫舰[J]. 飞航导弹, 2003(1): 27.

XU Wen. French Lafayette stealth frigate[J]. Winged Missiles Journal, 2003(1): 27 .

[5] 杨亚男, 郭建, 方雄伟. 舷侧排气系统在某船的应用[J]. 机电设备, 2015(4): 51-55.

YANG Yanan, GUO Jian, FANG Xiongwei. Application of side exhaust gas systems on one ship[J]. Mechanical and Electrical Equipment, 2015(4): 51-55.

[6] 袁江涛，杨立，陈睘羽，等. 现代舰船红外辐射及其控制策略分析 [J]. 激光与红外, 2006(10): 943-947.

YUAN Jiangtao, YANG Li, CHEN Xuan, et al. Analysis of infrared radiation on modern naval ship and its control strategies[J]. Laser \& Infrared, 2006(10): 943-947.

[7] 沐否, 王丽熙, 黄芸, 等. 红外隐身涂料的研究与发展趋势[J]. 材料
导报, 2017(1): 122-125.

MU Lei, WANG Lixi, HUANG Yun, et al. Research and development tendency of infrared stealthy coatings[J]. Materials Review, 2017(1): 122-125.

[8] 刘兵, 潘士兵, 于名汛, 等. 红外隐身涂料的研究与进展 $[\mathrm{J}]$. 兵器材 料科学与工程, 2017(5): 137-142.

LIU Bing, PAN Shibing, YU Mingxun, et al. Research and progress of infrared stealth coating[J]. Ordnance Material Science and Engineering, 2017(5): 137-142.

[9] 孟子晖，李仁玢，邱丽莉，等. 多波段兼容隐身用光子晶体研究进 展[J]. 兵工学报, 2019(1): 198-206.

MENG Zihui, LI Renbin, QIU LIli, et al. Research progress on photonic crystal multiband stealth material[J]. Acta Armamentarii, 2019(1): 198-206. 